\title{
Social Media as a New Trend in Sri Lankan Digital Journalism: A Surveillance
}

\author{
Selvarajah Thuseethan ${ }^{1} \&$ Shanmuganatha Vasanthapriyan ${ }^{1}$ \\ ${ }^{1}$ Department of Computing and Information Systmes, Sabaragamuwa University of Sri Lanka, Sri Lanka \\ Correspondence: Selvarajah Thuseethan, Department of Computing and Information Systems, Sabaragamuwa \\ University of Sri Lanka, Belihuloya, Sri Lanka, Tel: 94-77-753-5832. E-mail: svpriyan@gmail.com
}

$\begin{array}{lc}\text { Received: September 18, } 2014 & \text { Accepted: January 8, } 2015 \quad \text { Online Published: April 20, } 2015 \\ \text { doi:10.5539/ass.v11n10p86 } & \text { URL: http://dx.doi.org/10.5539/ass.v11n10p86 }\end{array}$

\begin{abstract}
In the last five years the rapid growth of social media that has been observed is indicative of its importance and its integration into the daily lives of many people in Sri Lanka. In parallel with this, there has also been considerable growth in digital journalism through the online medium. But the reachability of social media is very much high with comparing the traditional online mediums. As the result social media is converted as an online news media and overcomes the existing media in digital journalism. This pilot study explores the new trend in digital journalism with the presence of social media and carries out empirical research to understand the effectiveness of social media as a news medium and an effort has been made to propose expansion strategies of social media in maximizing the effectiveness in future digital journalism in the Sri Lankan context.
\end{abstract}

Keywords: social media, news media, pilot study, empirical research, digital journalism

\section{Introduction}

New incredible developments in the internet and its associated technologies are opening great opportunities for development in many low-income countries. As a result, developing countries are immediately adapting to new technologies to increase internet access in many areas, despite a huge number of barriers that stand in the way. Social media have made tremendous impact on online computing, by providing users opportunities to connect with others and generate enormous content on a daily basis. The enormous user participation in these social media is reflected with countless number of updates, opinion, news, comments and reviews being constantly posted and discussed in social web sites.

By considering the number of users and reachability the online news media overtakes the traditional media such as newspapers and televisions. While many online media remain important in news industry, online media primarily facilitate one-way news dissemination. Social media are conducive to timely, interactive communication and foster dialogue and content exchange among message consumers and creators (Seltzer \& Mitrook, 2007; Taylor \& Perry, 2005; Wright \& Hinson, 2009). As stated above social media can create opportunities for two-way interaction in between news providers and individuals in timely manner.

The use of social media is widely spread and common in developed countries where computing equipment, handhold devices, internet facility, and electricity are ever-present. Throughout this empirical research work, we examine influence of social media on digital journalism in a country where social media presence is ever growing, but less developed scientific infrastructures and irregular access to technology limit use. In this sense Sri Lanka is a developing island nation situated in the Indian Ocean southeast of India, is the 24th-largest island in the world. It is a low-income country where technologies are proliferating rapidly in all parts of the country, particularly in urban areas. In the Sri Lankan context the usage of social media increased rapidly during the last five years. Its mixed population, rich culture and variety of religions and languages reflect in the news industry. In this way the news industry is separated as culture wise, religion wise and language wise. Even though multiple separations exist, this research and the conclusions of it primarily focused on language wise separation.

Sri Lanka offers a perfect setting for this study in that it is one of the afresh emergent developing countries where the pace of development is medium. The social media and its associated technologies are opening new opportunities as well as challenges for development. This study is only a small, exploratory step and I admit that a new trend set by social media in Sri Lankan online news industry. There is a strong cooperative relationship 
has arisen between online news distribution and social media, social media provide us with a grip on the impact of news events, vice versa, much of what is conferred in social media is stimulated by the news.

\section{Aims and Objectives}

\subsection{Aims}

The significance and major purpose of the study was to reveals the importance of social media in this era and to establish and develop a better way of information dissemination in digital journalism with the help of ever evolving social media. Understanding of the influence of present social media and network technologies in developing the digital journalism helps the way to enhance and add value to digital journalism.

\subsection{Objectives}

Following sub-objectives were fulfilled to attain the overall purpose of the study, with the help of a questionnaire/survey, through which the data was analyzed:

- The determination of existing social media technologies, how those are categorized by the common people.

- Find out some factors influencing in use of social media

- To identify awareness or to what extent social media and network technology adapted in communities.

- Explore the view of community over the digital journalism.

- To suggest efficient social media representation to adapt digital journalism.

\section{Literature Review}

\subsection{Social Media}

The understanding of social media is impossible without first defining Web 2.0: a term that describes a new way in which end users use the World Wide Web, a place which contains dynamic contents continuously reformed by all operators in a sharing and joint way (Kaplan \& Haenlein, 2010). Social media has become increasingly popular among societies as a new technology that reaches out to many young people.

Social media consists of online technologies, practicing activities or societies that people use to generate content and share thoughts, visions, experiences and viewpoints with each other (Television Bureau of Advertising, Inc., 2009). In the sense the social media are the collection of interactive web pages that feature content users may generate, manipulate, or influence. Popular networks such as Facebook and Twitter are well-known globally and contain several hundreds of millions of users all over the world.

Social media tools such as Twitter, Facebook, blogs, RSS, and wikis (Kaplan \& Haenlein, 2010) are currently a part of most internet users' day to day communication (Boyd \& Ellison, 2007) and a large number of organizations have joined these online communities to stay connected to the public and members. These services can be commanding tools for information distribution, sharing, and creation and have a vital role to play in the future. But the term social media includes a variety of web and mobile based technologies ranging from photo and video sharing sites to rating and review forums.

\subsubsection{Who Uses What, and How?}

Social media marketer Michael Stelzner found through an online survey of more than 880 participants was steered in March of 2009. Most number of the partakers use social media marketing to some range, and the study pursued to reveal the "who, what, when, how, where and why" of social media usage (Stelzner, 2009). In recent years, micro blogs and social networking sites have become increasingly popular among online communities (Weerasinghe, Marasinghe, Ranaweera, Amarakeerthi, \& Cohen, 2013, July). Social media reaches more people than news media, and in Sri Lanka more people involve social media in one day than the cumulative weekly readership of every national newspaper in any medium. It is more popular than any online activity among active internet users. Facebook is the most popular social medium in Sri Lanka. Here we deal with social media users those who at least get connected with one of it.

\subsubsection{Categorization}

Social Media is a notoriously difficult area to categorize. In this research we categorized the social media into six broad categories.

- Social Network: It is a network of social collaboration and personal relationships, a web site on the internet that brings people with similar interest and background together in a centralized location to talk, exchange ideas, express interests, make new friendships, etc. Generally they consist of a profile, numerous ways to interact with others, ability to create groups, etc. The most popular are Facebook, LinkedIn and Google+, etc. 
- Bookmarking Site: It is a centralized service that allows people to save, organize and manage links to various websites and resources all over the internet. Most allow people to "tag" the links to make easy to search and share. Some of the most popular are Delicious and StumbleUpon.

- Social News: Services that allow people to post various news items or links and they are ranked based on the popularity. The community decides which news items get seen by more people. These sites are used to link many types of information including news. The most popular are Digg and Reddit.

- Media Sharing: It means the services that allow peoples to upload and give access to various media such as pictures, video and dynamic contents. Most services have additional social features such as likes, profiles, posts and commenting, etc. The most popular are YouTube and Flickr.

- Micro blogging: It means the services that concentrate on tiny updates that are published to anyone subscribed to accept the updates. The most popular example is Twitter.

- Blogs: Online forums let members to hold discussions by publishing/posting messages. Blog comments are alike except they are attached to blogs and usually the discussion centers around the topic of the blog post. There are numerous widespread blogs and forums but Google blogger is the best example.

\subsubsection{Reasons for Public Use Social Media}

Americans primarily use social media to maintain connections with family members, colleagues and friends, with roughly two-thirds reporting this as a leading reason for use (Austin, Liu, \& Jin, 2012; Pew Internet, 2011). However, the public's motivations for social media consumption somewhat vary, dependent on the need. The Pew Research Center report on "The State of News Media 2013" states that " $15 \%$ of US adults get their news primarily from social media, and the vast majority of them (77\%) follow links to consume the full news stories". Liu, Julia, and Jin (2012) collectively make known multiple reasons the public uses social media:

- Because of convenience

- Based on social norms

- Based on personal recommendations

- For humor \& levity

- For information seeking

- For timely information

- For unfiltered information

- To determine disaster magnitude

- To check in with family \& friends

- To self-mobilize

- To maintain a sense of community

- To seek emotional support \& healing

In terms of digital journalism information seeking, timely information and unfiltered information are related with the news contents spread over social media.

\subsubsection{Social Media in Sri Lanka}

After the end of thirty years of civil war Sri Lanka suddenly adapt to current and ongoing technology. In last few years the rapid growth in usage of social medium is extreme in Sri Lanka. Facebook as one of these social networks has become a popular social medium among contemporary Sri Lankan youth (Silva \& Peliarachchi).

\subsection{Digital Journalism}

Journalism is the practice of investigating, analyzing and reporting occasions, problems and trends to the mass spectators of print, broadcast and online media such as newspapers, posters, magazines, publications and books, radio and television stations and mass networks, and blogs and social and mobile media. Journalism may not necessarily want to engage in the social media revolution but they are being forced to as if they do not they would be left behind.

Journalism on digital networks, and specifically on Internet, is a relatively recent phenomenon, whose spread began in approximately 1994, in parallel to that of the World Wide Web. Study of this new communicative phenomenon began simultaneously in several countries. Digital journalism also known as online journalism is a 
contemporary form of journalism where editorial content is distributed via the Internet as opposed to publishing via printer broadcast. The primary product of journalism which is news and features on current affairs, is presented exclusively solely or as amalgamation of text, photos, audio, video and selected interactive forms, and distributed to become widely known through digital media platforms.

Digital journalism has also turned to social media as it is the new form of technology that will take precedence over other older forms of technology, it can no longer provide exposure to their news contents with the ancient way of dissemination. Social media and user generated content is increasingly moving centre stage; influencing the strategic direction and practice of journalism (Newman, 2009). News plays a varying role across the social networking sites. Approximately half of both Facebook and Twitter users acquire news on such social sites, previous reports have shown. On the other hand in YouTube, that is true of only one-fifth of its user base, and for LinkedIn, the number is even minor than previous.

\subsection{News Articles/News Posts}

A news article discusses current or recent news of either common concern (i.e. newspapers) or of an exact issue (i.e. magazines related with specific field, newsletters of any organization or public sectors, or news websites related with technology). We considered news articles/news posts in this research. News articles are very dynamic due to their relation to continuously developing events that typically have short lifespans. For a news article to be popular, it is essential for it to propagate to a large number of readers within a short time.

\section{Research Methodology}

In this study, prime data was collected from common peoples from Sri Lanka which comprises both males and females, with a questionnaire comprising both closed-ended and open-ended questions. The selected people are the users of least one social media component. At the same time data collected from Sri Lankan famous news websites also used in this research. In nature the design of research include both cross-sectional and exploratory. Quantitative and qualitative approaches imposed in primary data collection to carry out the research work. In this study, to formulate the questionnaire the literature was used as the source of information. In planning our survey, we aimed for a few number of direct, multiple choice questions which would serve to reveal norms and also contain some one word answer question. To increase the ability in answering the questionnaire the respondents instructed by numerous instructions throughout the questionnaire, and each and every questionnaire was personally cared and administered. To standardize the surroundings under which the questionnaires were accomplished; research assistants at local villages in the selected areas and representatives from each website were recruited and trained as co-workers. Finally all the details gathered and analyzed carefully with the help of analytical tools.

\section{Results and Discussion}

\subsection{User's Perspective}

The survey conducted nationwide among the Sri Lankan adult users which include 1000 respondents. They are divided in to groups with respect to above broad categories of social media. Among the sample users $74 \%$ of them using social networks, $11 \%$ of them using media sharing, $9 \%$ users using micro blogging and $2 \%$ users using blogs, micro blogging and social news. The following chart describes the sample sizes for users on each broad category. Figure 1 shows the overall distribution of selected peoples in terms of usage of each category.

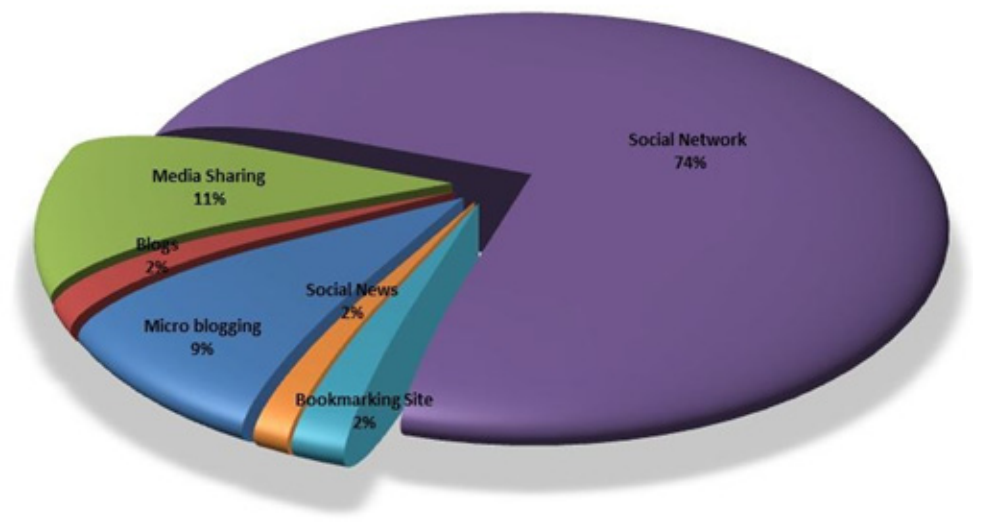

Figure 1. Distribution of users in each social media category 
Further we analyzed the reason for the pattern of user distribution in major classifications. Several reasons were there behind vast usage of social network. The first three reasons are;

- Crazy about Facebook

- Looking forward to news and/or interesting posts

- For professional purposes

Second most reason to use social networking sites is the gateway to access news materials and other interesting posts provided by several news sites. John S. and James L. Knight Foundation with the support of Pew Research Center collectively found in Facebook, the leading social media platform; news is a general experience. Our research also indicates that commonly most number of users use Facebook to get access of news materials. The increasing significance of Facebook recommendations are reinforced by the survey outcomes.

The candidates who wish to get access of news content through social networking also suggest the mode to represent those in main page. In that survey question $94 \%$ candidates prefer either a separate portal or separate grouping in home page to access news contents. Figure 2 shows the complete result.

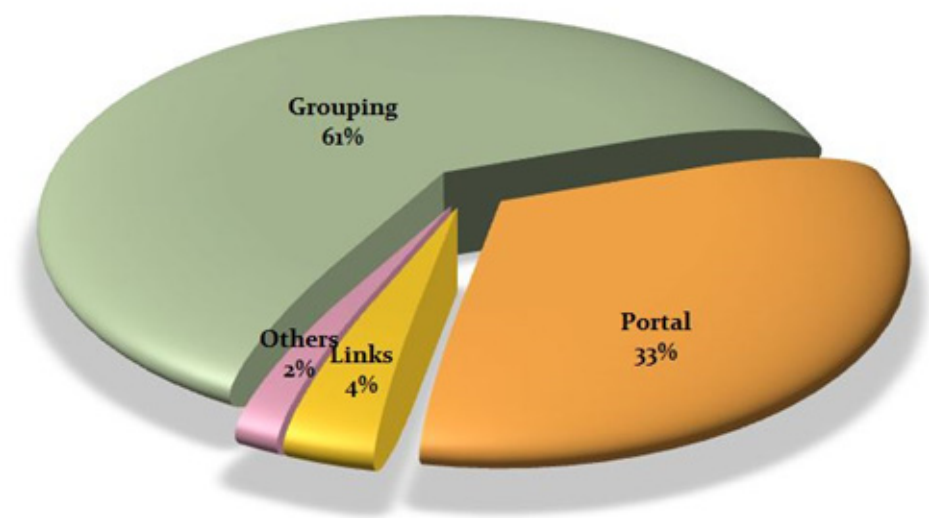

Figure 2. Modes of presenting news contents in social network: user's perspective

\subsubsection{Likes and Dislikes of Viewing News through Social Media}

Among those candidates $91 \%$ of them like to view news articles through their social media pages. Remaining $9 \%$ candidates strongly dislike the existence of news links in social media pages. Figure 3 shows the result.

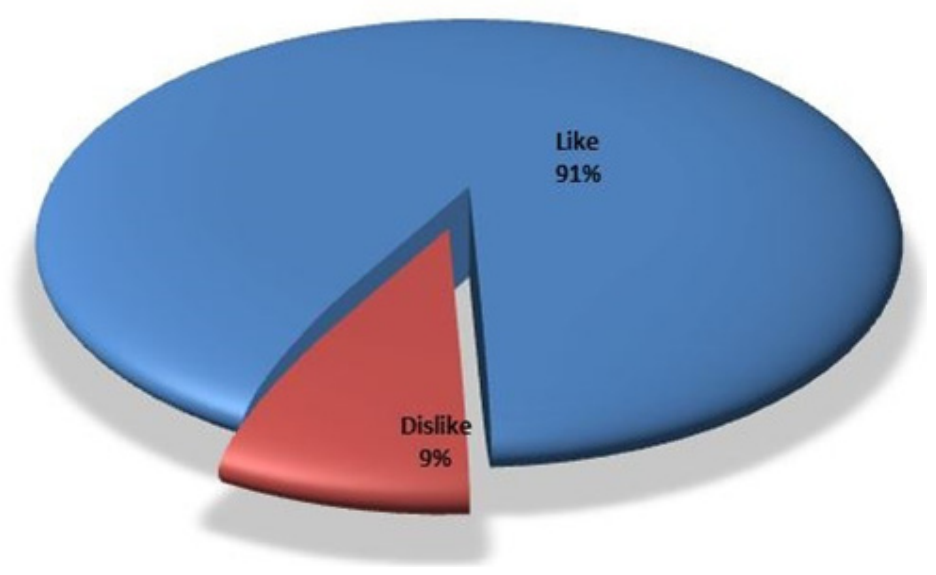

Figure 3. Existence of news links in social media pages: Likes and dislike

Since the majority of candidates fall into social network category we analyzed them separately. The result we revealed is all the candidates like to view news contents through that. At the same time $82 \%$ in other category users also suggest social network as the best place to disseminate news articles with comparing other categories. 


\subsubsection{The Users Overlap}

The majority of users using more than one social medium and they fall into many categories. For those who fall in more than one category, social network is likely to be one of the ones they use. More than half of the Sri Lankan adults who uses media sharing, blogs, micro blogging, social news and bookmarking site also using social network. Aside from that, the intersect users between other categories is relatively small.

\subsection{Digital News Sites Perspective}

We took 5 famous local news websites in Sri Lanka for our research work and all of them are maintaining at least one social medium. In that sample 2 news web sites providing their news contents in three languages such as Sinhala, Tamil and English. Other 2 websites provide news only in Sinhala. Other 1 website provides news contents only in Tamil.

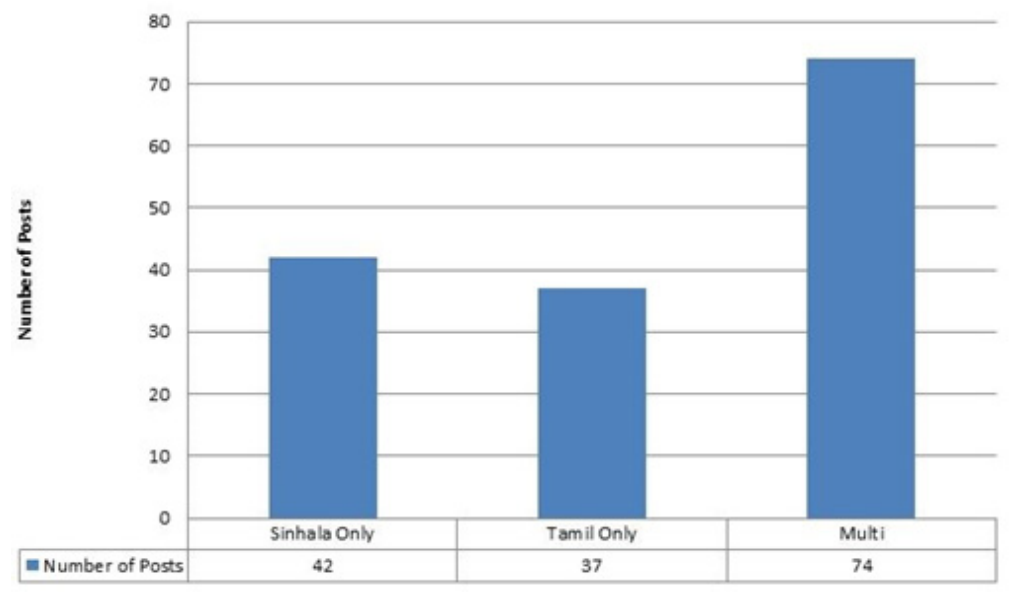

Figure 4. Average Number of posts published in news sites

We categorize those news sites into three broad categories as Sinhala language sites, Tamil language sites and multi-language sites. The average news posts posted in a day by each category news mediums are given in the Figure 4. The hits/views per day in each category news mediums are given in Figure 5. Since we fully concentrate on Sri Lankan perspective only consider the local views/hits in our research

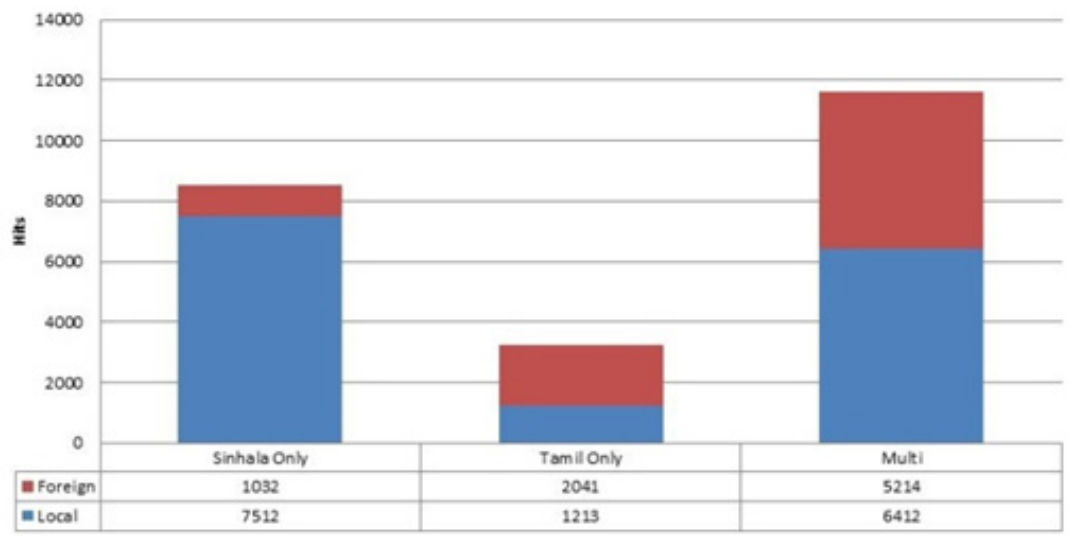

Figure 5. Average hits/views per day by local and foreign candidates

As main part of the interviews with candidates we asked broadly about their present use of social media in receiving and viewing news articles. But here we got the amount of actual hits through social media. In all three mediums half amount of candidates went through social media to view news contents published by news sites. Figure 6 shows the complete results 


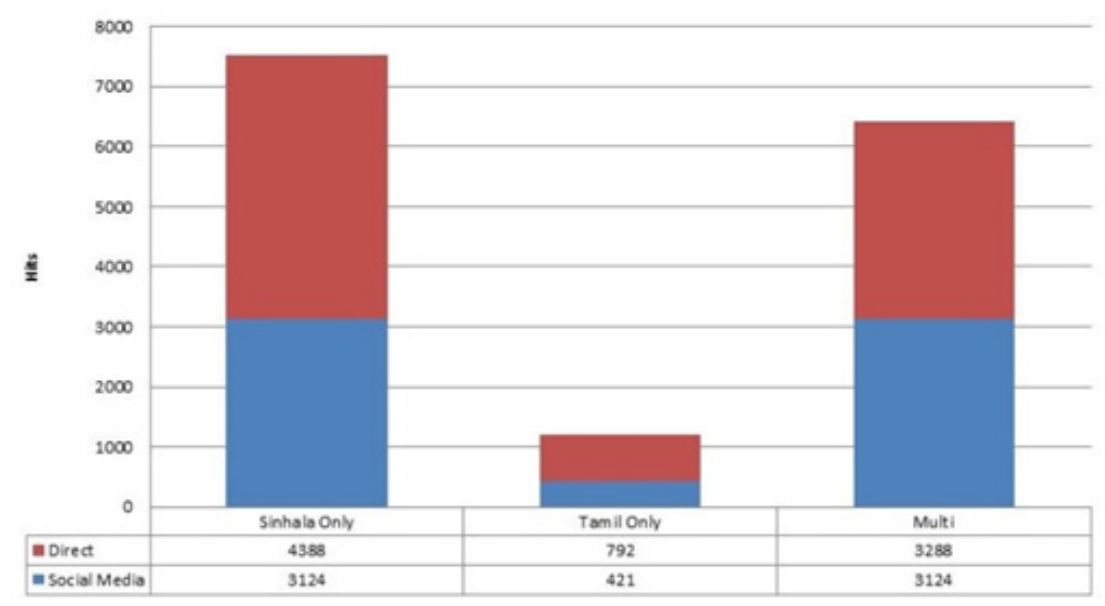

Figure 6. Hits or views to news contents: Direct Vs. through social media

\section{Conclusion}

Social media surveillance is a comparatively novel form of surveillance that is centered on making evident on convergence of social characteristics and social accomplishments to peoples. As we spend more and more time online on social media, a lot of our routine activities in different roles during a lot of our working and free time become analyzable, accessible and traceable.

Social media play a major role in dissemination of news articles. Most of the people use social media as main medium to get access of news articles. They suggest a different portal or separate grouping in broadcasting or publishing news articles in social media. At the same time social networking category leads in promoting digital journalism.

\section{References}

Bandari, R., Asur, S., \& Huberman, B. A. (2012, February). The Pulse of News in Social Media: Forecasting Popularity. In ICWSM.

Ellison, N. B. (2007). Social network sites: Definition, history, and scholarship. Journal of Computer-Mediated Communication, 13(1), 210-230. http://dx.doi.org/10.1111/j.1083-6101.2007.00393.x

Flew, T. (2005). New media: An introduction. Oxford University Press.

Jones, H., \& Soltren, J. H. (2005). Facebook: Threats to privacy. Project MAC: MIT Project on Mathematics and Computing, 1 .

Kwak, H., Lee, C., Park, H., \& Moon, S. (2010, April). What is Twitter, a social network or a news media? In Proceedings of the 19th international conference on World wide web (pp. 591-600). ACM. http://dx.doi.org /10.1145/1772690.1772751

Lariscy, R. W., Avery, E. J., Sweetser, K. D., \& Howes, P. (2009). An examination of the role of online social media in journalists' source mix. Public Relations Review, 35(3), 314-316. http://dx.doi.org/10.1016/j. pubrev.2009.05.008

Matthews, L. (2010). Social media and the evolution of corporate communications. The Elon Journal of Undergraduate Research in Communications, 1(1), 17-23.

Morris, M. R., Teevan, J., \& Panovich, K. (2010, April). What do people ask their social networks, and why? A survey study of status message q\&a behavior. In Proceedings of the SIGCHI conference on Human factors in computing systems (pp. 1739-1748). ACM. http://dx.doi.org/10.1145/1753326.1753587

Thuseethan, S., \& Kuhanesan, S. (2014). Influence of Facebook in Academic Performance of Sri Lankan University Students. Global Journal of Computer Science and Technology, 14(4). http://dx.doi.org/10.2139/ ssrn.2478336

Tsagkias, M., de Rijke, M., \& Weerkamp, W. (2011, February). Linking online news and social media. In Proceedings of the fourth ACM international conference on Web search and data mining (pp. 565-574). 
ACM. http://dx.doi.org/10.1145/1935826.1935906

van der Haak, B., Parks, M., \& Castells, M. (2012). The future of journalism: Networked journalism. International Journal of Communication, 6, 16.

Weerasinghe, P., Marasinghe, A., Ranaweera, R., Amarakeerthi, S., \& Cohen, M. (2013, July). Emotion Expression for Affective Social Communication. In Biometrics and Kansei Engineering (ICBAKE), 2013 International Conference on (pp. 148-153). IEEE.

Wyche, S. P., Schoenebeck, S. Y., \& Forte, A. (2013, February). Facebook is a luxury: An exploratory study of social media use in rural Kenya. In Proceedings of the 2013 conference on Computer supported cooperative work (pp. 33-44). ACM. http://dx.doi.org/10.1145/2441776.2441783

\section{Copyrights}

Copyright for this article is retained by the author(s), with first publication rights granted to the journal.

This is an open-access article distributed under the terms and conditions of the Creative Commons Attribution license (http://creativecommons.org/licenses/by/3.0/). 